\title{
An Empirical Three-Dimensional Crossover Capacitance Model for Multilevel Interconnect VLSI Circuits
}

\author{
Shyh-Chyi Wong, Trent Gwo-Yann Lee, Dye-Jyun Ma, and Chuan-Jane Chao
}

\begin{abstract}
We develop an empirical model for the crossover capacitance induced by the wire crossings in VLSI with multilevel metal interconnects. The crossover capacitance, which is formed in any three adjacent layers and of a three-dimensional (3-D) nature, is derived in closed form as a function of the wire geometry parameters. The total capacitance on a wire passing many crossings can then be easily determined by combining the crossover capacitance with the two-dimensional (2-D) intralayer coupling capacitance defined on a same layer. The model agrees well with the numerical field solver (with a $6.7 \%$ root-mean-square error) and measurement data (with a maximum error of $4.17 \%$ ) for wire width and spacing down to $0.16 \mu \mathrm{m}$ and wire thickness down to $0.15 \mu \mathrm{m}$. The model is useful for VLSI design and process optimization.
\end{abstract}

Index Terms-Closed-form models, crossover capacitance, multilevel interconnects, VLSI circuits.

\section{INTRODUCTION}

D EEP submicrometer integrated circuit performance is influenced by interconnect RC delay [1]-[3]. Although the device delay decreases as the technology scales down, the interconnect-induced delay, however, increases, because both line resistance and intralayer capacitance increase [1], [2], [4]. In VLSI circuits with multilevel interconnects, lines in adjacent metal layers are placed orthogonally to each other to minimize overlapped capacitances and enhance routing flexibility. This procedure forms many wire crossings, inducing crossover capacitance, which becomes the major factor in affecting the circuit speed [2], [5]. An accurate model for the crossover capacitance is essential for estimating the interconnect circuit performance.

Many previous works on interconnects exist in the literature. The works of [3], and [5]-[7] either considered two-dimensional (2-D) structures or approximated the three-dimensional (3-D) wirings by 2-D cross sections; both approaches cannot model 3-D fringe field. The models of [5]-[7] were based on numerical solutions, thus not allowing for closed-form estimation. The work of Chern [2] gave a crossover model for triple-level metal layers but with same thickness in all layers. The work of

Manuscript received May 8, 1999; revised November 15, 1999.

S.-C. Wong is with the Research Development, Taiwan Semiconductor Manufacturing Corporation, Hsinchu, Taiwan, ROC.

T. G.-Y. Lee is with the Institute of Electronics Engineering, National Chiao-Tung University, Hsinchu, Taiwan, ROC.

D.-J. Ma is with the Department of Electrical Engineering, National ChungHsing University, Taichung, Taiwan, ROC.

C.-J. Chao is with the Institute of Electronics Engineering, National Chiao-Tung University, Hsinchu, Taiwan, ROC.

Publisher Item Identifier S 0894-6507(00)03546-6.
Pan et al. [8] derived an analytical expression for crossover capacitance specifically for packaging geometries. The work of Kuhn et al. [9] gave an optimization study for delay time and power dissipation using combined device and interconnect capacitances; it, however, ignored both intralayer coupling and crossover capacitances that are important in deep submicron VLSI. The work of Vladimir and Mittra [10] gave improved boundary conditions for numerical solution of interconnect and packaging capacitances. Some other works focused on novel measurement methods for extracting interconnect capacitance on various layout structures. For instance, the work of Wee $e t$ al. [11] developed a complete set of structures for characterizing multilevel metal capacitances for both stack and crossing configurations; the impact of metal-edge slope and void was also extracted. The work of Nouet and Toulouse [12] characterized interlayer and intralayer capacitance novel test patterns, and compared on-chip and off-chip measurement. In [12], it was identified that the 3-D crossings (crossover) is a critical component in the total wiring capacitance, and a linear model with different components was then proposed with linear dependence on area, periphery length, and spacing. The work of Aoyama et al. [13] characterized coupling and ground capacitance using test patterns and numerical solutions, and it provided an optimization study by wire pitch to dielectric thickness ratio. The work of Chao et al. [14] presented a novel extraction methodology and test pattern, with verifications on SOG and CMP processes. The work of Chen et al. [15] gave a novel on-chip measurement method for small wire capacitance. In [16], we developed models for 2-D wiring capacitance, wire delay, and interwire cross-talk noise. The capacitance model of [16] gives accurate intralayer and line-to-ground capacitance estimation for both parallel lines on a plane and lines between two planes, with agreement with measurement data.

In this paper, we continue our previous effort of [16] by focusing on the modeling of crossover capacitance for VLSI's with multilevel metal interconnect of arbitrary dielectric and wire thickness, width, and spacing in all layers. The crossover capacitance is formed in any three adjacent layers of the multilevel metal interconnects and is of a 3-D nature. We derive closed-form formula for the crossover capacitance as a function of the wire geometry parameters of three adjacent layers, including the wire width, spacing, thickness, and dielectric thickness of a line and of lines in the upper and lower layers. The total net capacitance on a wire passing many crossings can then be easily obtained by combining the crossover capacitance with the 2-D intralayer coupling capacitance defined on a same layer 
obtained in [16]. The result of our model shows agreement with the numerical field solver [17] and measurement data. This work extends the work of [16] to provide a complete solution for the modeling of interconnect capacitance for arbitrary multilevel interconnects. The complete model can be used in the delay and capacitance estimation in circuit design and process optimization.

\section{CAPAcitance Model}

The crossover capacitance is formed in any three adjacent layers of the multilevel metal interconnect. Consider any triplelevel wire crossings, as shown in Fig. 1, where the second-level metal lines $\left(M_{2}\right)$ cross the first-level $\left(M_{1}\right.$, the lower level $)$ and third-level $\left(M_{3}\right.$, the upper level) metal lines. The line width, spacing, and thickness are denoted by $W_{i}, S_{i}$, and $T_{i}$ for the $i$ th-level metal layer, $i=1,2,3$. The dielectric layer thickness is denoted by $H_{1}, H_{2}$, and $H_{3}$ for the dielectric between $M_{1}$ and the substrate (or the next lower layer, say, $M_{0}$ ), $M_{2}$ and $M_{1}$, and $M_{3}$ and $M_{2}$, respectively. For each $M_{2}$ line crossing $M_{1}$ line, a crossover capacitance $C_{c r}$ exists. Note that $M_{1}$ and $M_{3}$ lines are not necessarily aligned to each other. This capacitance $C_{c r}$ is restricted within a neighborhood of the lines intersection. Outside the intersection neighborhood, $M_{2}$ line capacitance can be estimated by existing 2-D intralayer coupling capacitance models [16].

To derive the crossover model for $C_{c r}$, we adopt an empirical approach here, because the usual power series or numerical solutions for Poisson's equation are not appropriate for VLSI simulation [17], [18]. In deriving these expressions, a rational function is first constructed to model each type of electrical flux variations with geometry variation. The rational functions are then multiplied to each other to form one flux component. Finally, all flux components are added, giving the lumped crossover capacitance $C_{c r}$. Here, three flux components, $C_{1}, C_{2}$, and $C_{3}$, are involved. That is

$$
C_{c r}=C_{1}+C_{2}+C_{3} .
$$

Capacitance $C_{1}$ represents the area component from $M_{1}$ top surface to $M_{2}$ bottom surface. Capacitance $C_{2}$ represents the component from $M_{1}$ side wall to $M_{2}$ bottom surface. Capacitance $C_{3}$ represents the component from the $M_{2}$ side wall to $M_{1}$ top surface.

To derive $C_{1}$, note that $C_{1}$ is simply the plate-to-plate capacitance, and hence

$$
\frac{C_{1}}{\epsilon_{\circ x}}=\frac{W_{1} W_{2}}{H_{2}} .
$$

The flux component $C_{2}$ is modeled as the product of rational functions in the following general form:

$$
\begin{aligned}
\frac{C_{2}}{\epsilon_{0 x}}= & c_{1} W_{2}^{\alpha_{1}}\left(S_{1} \times S_{2}\right)^{\alpha_{2}}\left(\frac{T_{1}}{T_{1}+c_{2} H_{2}}\right)^{\alpha_{3}}\left(\frac{T_{1}}{T_{1}+c_{3} S_{1}}\right)^{\alpha_{4}} \\
& \times\left(\frac{H_{1}}{H_{1}+c_{4} S_{1}}\right)^{\alpha_{5}} \exp \left(\frac{-H_{2}}{c_{5}\left(S_{1}+c_{6} H_{2}\right)}\right)
\end{aligned}
$$

where the $c_{i}$ 's are constants and the $\alpha_{i}$ 's are the power coefficients, both to be determined later.
We now explain the physical rationale behind each term adopted on the right-hand side of (3) for $C_{2}: 1$ ) the $W_{2}$ term follows from a power-law dependence of the capacitance on the line width [2], [3], [18];2) the $S_{1}$ and $S_{2}$ terms are to catch the intrawire spacing dependence: Because the $M_{1}$ side wall to $M_{2}$ wire flux is reduced by intra- $M_{1}$ flux as shown in the cross-section A of Fig. 1, $C_{2}$ decreases with reduced intra- $M_{1}$ spacing $S_{1}$; similar impact can be induced by intra- $M_{2}$ spacing $S_{2}$. Here, the same power coefficient $\alpha_{2}$ is used to reflect their same influence; 3$)$ the term $\left(T_{1} /\left(T_{1}+c_{2} H_{2}\right)\right)^{\alpha_{3}}$ is adopted to model the fact that the flux originated from side wall heavily relies on the wire side wall thickness with a power-law dependence [3]. The power-law dependence has been proved in [18] as a good approximation to the field strength between adjacent nonoverlapping perpendicular surfaces. Note that this dependence will be weakened for large thickness (because such flux only exists at the side wall corner adjacent to the dielectric layer); the constant $c_{2}$ reflects this dependence weakening; 4) the terms $\left(T_{1} /\left(T_{1}+c_{3} S_{1}\right)\right)^{\alpha_{4}}$ and $\left(H_{1} /\left(H_{1}+c_{4} S_{1}\right)\right)^{\alpha_{5}}$ are used to model the fact that $C_{2}$ decreases with reduced $T_{1} / S_{1}$ as well as with reduced $H_{1} / S_{1}$ because of enhanced flux from $M_{1}$ to ground plane, as shown in the cross-section A of Fig. 1; and 5) the exponential term modifies the $1 / H_{2}$ dependence constructed in (2), giving weakened $\mathrm{H}_{2}$ impact with increased $H_{2} / S_{1}$, because intra- $M_{1}$ flux prevents field lines from being pulled up to $M_{2}$, electrode, as shown in the cross-section A of Fig. 1.

To derive $C_{3}$, we observe that $C_{3}$ is approximately a $180^{\circ}$ turnover of $\mathrm{C}_{2}$. Therefore, similar mathematical patterns will be adopted to emulate the similar electrical flux distributions. Differences in $C_{2}$ and $C_{3}$ exist, however: $C_{2}$ has a larger plate next to (or under) the side wall flux, whereas $C_{3}$ has many narrower wirings ( $M_{3}$ wires) next to (or above) the side wall component. The consequence is that the side wall flux reduction induced by larger adjacent plane in $C_{2}$ and by adjacent wirings in $C_{3}$ will be different. $C_{3}$ is modeled in the following general form:

$$
\begin{aligned}
\frac{C_{3}}{\epsilon_{o x}}= & d_{1} W_{1}^{\beta_{1}} S_{1}^{\beta_{2}} S_{2}^{\beta_{3}}\left(\frac{T_{2}}{T_{2}+d_{2} H_{2}}\right)^{\beta_{4}} \exp \left(\frac{-H_{2}}{d_{3}\left(S_{2}+d_{4} H_{2}\right)}\right) \\
& \times\left(\frac{H_{3}}{H_{3}+d_{5} S_{2}}\right)^{\beta_{5}}
\end{aligned}
$$

where the $d_{i}$ 's are constants and the $\beta_{i}$ 's are the power coefficients, both again to be determined later.

Now, similar physical explanation, as is the case with $C_{2}$, can be made for each term on the right-hand side of (4) for $\left.C_{3}: 1\right)$ the $W_{1}$ term shows the power-law dependence as before; 2 ) the power terms of $S_{1}$ and $S_{2}$ again catch the intrawire spacing dependence, but here we use different power coefficients for them because their influence will be different. In fact, the influence of $S_{2}$ term in $C_{3}$ is weaker than in $C_{2}$, for the impact in $C_{3}$ is weakened by the $M_{3}$-to- $M_{2}$ flux; 3 ) the term $\left(T_{2} /\left(T_{2}+d_{2} H_{2}\right)\right)^{\beta_{4}}$ models the fact that $C_{3}$ increases with increased $\left.\mathrm{T}_{2} / \mathrm{H}_{2} ; 4\right)$ the exponential term further modifies the $1 / H_{2}$ dependence constructed in (2), giving weakened $H_{2}$ impact with increased $H_{2} / S_{2}$, because intralayer flux prevents field lines from being pulled down to $M_{1}$ electrode; and 5) the last term $\left(H_{3} /\left(H_{3}+d_{5} S_{2}\right)\right)^{\beta_{5}}$ models the impact of $M_{3}$ layer 


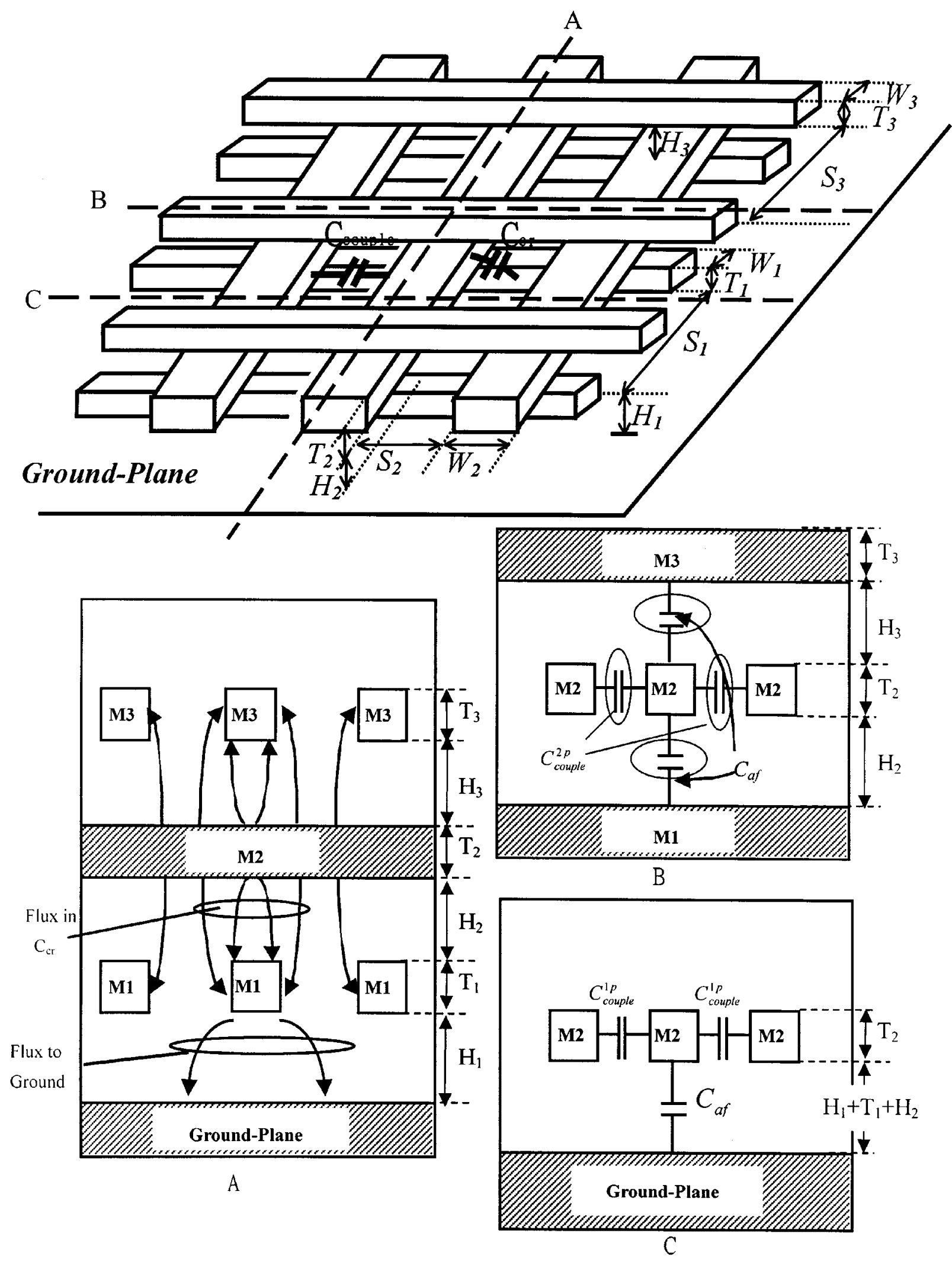

Fig. 1. Metal wiring crossover structure and cross sections along cut lines A, B, and C. $H_{1}, H_{2}$, and $H_{3}$ are dielectric thickness, $T_{1}, T_{2}$, and $T_{3}$ are metal wire thickness, $W_{1}, W_{2}$, and $W_{3}$ are wire width, and $S_{1}, S_{2}$, and $S_{3}$ are interwire spacing.

on $C_{3}$, which gives reduced $C_{3}$ with reduced $H_{3} / S_{2}$, because the intralayer coupling flux between $M_{2}$ lines forms a shield that isolates the $C_{3}$ flux from the influence of the $M_{3}$-to- $M_{2}$ flux.
This shielding effect is very strong when $S_{2}$ is small, as shown in the cross-section B of Fig. 1. This shielding effect is reduced with large $S_{2}$, and hence, $C_{3}$ can be significantly reduced with 
reduced $H_{3}$. Note that this effect is opposed to the phenomenon that $C_{3}$ increases with increased $S_{2}$, as predicted by the power terms of $\left(S_{1}\right)^{\beta_{2}}$ and $\left(S_{2}\right)^{\beta_{3}}$. This term and the power-law term provide contradictory influences by $S_{2}$ spacing, and our model can well describe these two opposing phenomena, which will later be demonstrated in Fig. 3.

To determine all constants and power coefficients in (3) and (4) for $C_{2}$ and $C_{3}$, we use the approach of least-mean-squareserrors fitting, and we obtain

$$
\begin{aligned}
\frac{C_{2}}{\epsilon_{o x}}= & 3.73 W_{2}^{0.6}\left(S_{1} \times S_{2}\right)^{0.2} \times\left(\frac{T_{1}}{T_{1}+0.035 H_{2}}\right)^{0.64} \\
& \times\left(\frac{T_{1}}{T_{1}+0.851 S_{1}}\right)^{0.12} \times\left(\frac{H_{1}}{H_{1}+0.051 S_{1}}\right)^{1.0} \\
& \times \exp \left(\frac{-H_{2}}{0.7\left(S_{1}+0.4 H_{2}\right)}\right)
\end{aligned}
$$

and

$$
\begin{aligned}
\frac{C_{3}}{\epsilon_{O x}}= & 3.73 W_{1}^{0.6} S_{1}^{0.2} S_{2}^{0.1}\left(\frac{T_{2}}{T_{2}+0.035 H_{2}}\right)^{0.64} \\
& \times \exp \left(\frac{-H_{2}}{0.7\left(S_{2}+0.4 H_{2}\right)}\right) \times\left(\frac{H_{3}}{H_{3}+0.015 S_{2}}\right)^{3} .
\end{aligned}
$$

The root-mean-square error between the model and the numerical solutions is $6.71 \%$, based on a total of 272 data points using the least-squares-error fitting approach. The 272 total data points were basically selected randomly, but with more dense data points chosen toward smaller dimension range (as the capacitance effect is more pronounced at smaller dimension range). A list of error distribution is shown is Table I, which only displays a partial set of our data used for parameter fitting.

The segments of $M_{2}$ outside the intersection neighborhood can be modeled by the 2-D capacitance formulas derived previously by the authors [16]. The capacitance components here include 1) intralayer coupling capacitance $C_{\text {couple, }}^{1 p}$, which is the intra- $M_{2}$ flux in the wire region without $M_{3}$ wirings crossing above, as shown in the cut-line $\mathrm{C}$ and cross section $\mathrm{C}$ of Fig. 1, 2) $C_{\text {couple }}^{2 p}$, which is the intra- $M_{2}$ flux in the $M_{2}$ region with $M_{3}$ wirings crossing above, as shown in the cut-line $\mathrm{B}$ and cross-section B of Fig. 1, and 3) line-to-ground capacitance $C_{a f}$ in the region without $M_{3}$ wirings crossing above, as shown in the cross section C of Fig. 1. These capacitances were obtained in [16] as

$$
\begin{aligned}
\frac{C_{a f}}{\epsilon_{o x}}= & \frac{W_{2}}{H}+2.217\left(\frac{S_{2}}{S_{2}+0.702 H}\right)^{3.913} \\
& +1.171\left(\frac{S_{2}}{S_{2}+1.51 H}\right)^{0.764} \\
& \times\left(\frac{T_{2}}{T_{2}+4.532 H}\right)^{0.12} \\
\frac{C_{\text {couple }}^{1 p}}{\epsilon_{\text {ox }}}= & 1.144 \frac{T_{2}}{S_{2}}\left(\frac{H}{H+2.059 S_{2}}\right)^{0.0944} \\
& +0.7428\left(\frac{W_{2}}{W_{2}+1.592 S_{2}}\right)^{1.144}
\end{aligned}
$$

\begin{tabular}{|c|c|c|c|c|c|c|c|c|c|c|c|c|}
\hline \multicolumn{6}{|c|}{ Technology dimension $(\mu \mathrm{m})$} & \multicolumn{6}{|c|}{ Wire dimension $(\mu \mathrm{m})$} & \multirow[t]{2}{*}{ Error(\%) } \\
\hline H3 & & HI & $\mathbf{T 3}$ & $\mathbf{T 2}$ & T1 & W3 & W2 & W1 & $\mathbf{S 3}$ & $\mathbf{S 2}$ & S1 & \\
\hline 2.0 & 2.0 & 2.0 & 2.0 & 2.0 & $\frac{1}{2.0}$ & 2.0 & 2.0 & $\frac{1}{2.0}$ & 2.0 & 2.0 & 2.0 & -2.700 \\
\hline 0.16 & 0.16 & 0.16 & 0.16 & 0.16 & 0.16 & 2.0 & 2.0 & 2.0 & 2.0 & 2.0 & 2.0 & -3.900 \\
\hline 0.89 & 0.89 & 0.89 & 0.64 & 0.64 & 0.64 & 0.8 & 0.8 & 0.8 & 1.0 & 1.0 & 0.16 & -2.285 \\
\hline 0.89 & 0.89 & 0.89 & 0.64 & 0.64 & 0.64 & 0.8 & 0.8 & 0.8 & 1.0 & 1.0 & 0.5 & 1.166 \\
\hline 0.89 & 0.89 & 0.89 & 0.64 & 0.64 & 0.64 & 0.8 & 0.8 & 0.8 & 1.0 & 0.16 & 1.0 & -3.982 \\
\hline 0.89 & 0.89 & 0.89 & 0.64 & 0.64 & 0.64 & 0.8 & 0.8 & 0.8 & 0.16 & 1.0 & 1.0 & -0.152 \\
\hline 0.89 & 0.89 & 0.89 & 0.64 & 0.64 & 0.64 & 0.8 & 0.8 & 0.8 & 1.0 & 1.0 & 1.0 & -0.223 \\
\hline 3.0 & 0.9 & 0.16 & 0.6 & 0.6 & 0.6 & 0.3 & 0.3 & 0.3 & 0.3 & 0.3 & 0.3 & 2.953 \\
\hline 3.0 & 0.9 & 3.0 & 0.6 & 0.6 & 0.6 & 0.3 & 0.3 & 0.3 & 0.3 & 0.3 & 0.3 & -7.232 \\
\hline 0.9 & 0.16 & 0.9 & 0.6 & 0.6 & 0.6 & 0.3 & 0.3 & 0.3 & 0.3 & 0.3 & 0.3 & 0.596 \\
\hline 0.16 & 0.3 & 0.3 & 0.3 & 0.3 & 0.3 & 0.3 & 0.3 & 0.3 & 0.3 & 0.3 & 0.3 & 2.682 \\
\hline 0.89 & 0.3 & 0.3 & 0.3 & 0.3 & 0.3 & 0.3 & 0.3 & 0.3 & 0.3 & 0.3 & 0.3 & 4.693 \\
\hline 3.0 & 0.3 & 0.3 & 0.3 & 0.3 & 0.3 & 0.3 & 0.3 & 0.3 & 0.3 & 0.3 & 0.3 & 5.145 \\
\hline 0.16 & 0.16 & 0.16 & 0.16 & 0.16 & 0.16 & 2.0 & 2.0 & 2.0 & 0.16 & 0.16 & 0.16 & -5.500 \\
\hline 0.3 & 0.3 & 0.3 & 0.3 & 0.3 & 0.3 & 0.16 & 0.16 & 0.16 & 50.16 & 0.16 & 0.16 & 8.905 \\
\hline 0.5 & 0.5 & 0.4 & 0.3 & 0.3 & 0.3 & 0.16 & 0.16 & 0.16 & 0.16 & 0.16 & 0.16 & 5.470 \\
\hline 0.5 & 0.5 & 0.9 & 0.3 & 0.3 & 0.3 & 0.16 & 0.16 & 0.16 & 0.16 & 0.16 & 0.16 & -0.241 \\
\hline 0.5 & 0.16 & 0.5 & 0.3 & 0.3 & 0.3 & 0.16 & 0.16 & 0.16 & 0.16 & 0.16 & 0.16 & 8.028 \\
\hline 0.16 & 0.6 & 0.6 & 0.3 & 0.3 & 0.3 & 0.16 & 0.16 & 0.16 & 0.16 & 0.16 & 0.16 & 0.414 \\
\hline 0.4 & 0.6 & 0.6 & 0.3 & 0.3 & 0.3 & 0.16 & 0.16 & 0.16 & 0.16 & 0.16 & 0.16 & 1.341 \\
\hline 0.89 & 0.6 & 0.6 & 0.3 & 0.3 & 0.3 & 0.16 & 0.16 & 0.16 & 0.16 & 0.16 & 0.16 & 1.759 \\
\hline 3.0 & 0.6 & 0.6 & 0.3 & 0.3 & 0.3 & 0.16 & 0.16 & 0.16 & 0.16 & 0.16 & 0.16 & 2.035 \\
\hline 0.16 & 0.16 & 0.16 & 2.0 & 2.0 & 0.16 & 0.16 & 0.16 & 0.16 & 0.16 & 0.16 & 0.16 & 4.800 \\
\hline 2.0 & 2.0 & 2.0 & 0.16 & 0.16 & 0.16 & 0.16 & 0.16 & 0.16 & 0.16 & 0.16 & 0.16 & 8.300 \\
\hline 2.0 & 0.16 & 0.16 & 0.16 & 0.16 & 0.16 & 0.16 & 0.16 & 0.16 & 0.16 & 0.16 & 0.16 & 6.300 \\
\hline 0.16 & 0.16 & 0.16 & 0.16 & 0.16 & 0.16 & 0.16 & 0.16 & 0.16 & 0.16 & 0.16 & 0.16 & 6.400 \\
\hline
\end{tabular}

TABLE I

ERRor TABLE OF THE MODEL COMPARED WITH NUMERICAL SOLUTIONS

$$
\begin{aligned}
& +1.158\left(\frac{W_{2}}{W_{2}+1.874 S_{2}}\right)^{0.1612} \\
& \times\left(\frac{H}{H+0.9801 S_{2}}\right)^{1.179}
\end{aligned}
$$

and

$$
\begin{aligned}
\frac{C_{\text {couple }}^{2 p}}{\epsilon_{\text {ox }}}= & 1.412 \frac{T_{2}}{S_{2}} \exp \left(-\frac{2 S_{2}}{S_{2}+8.014 H_{2}}-\frac{2 S_{2}}{S_{2}+8.014 H_{3}}\right) \\
& +1.1852\left(\frac{W_{2}}{W_{2}+0.3078 S_{2}}\right)^{0.25724} \\
& \times\left\{\left(\frac{H_{2}}{H_{2}+8.961 S_{2}}\right)^{0.7571}\right. \\
& \left.+\left(\frac{H_{3}}{H_{3}+8.961 S_{2}}\right)^{0.7571}\right\} \\
& \times \exp \left(-\frac{2 S_{2}}{S_{2}+3\left(H_{2}+H_{3}\right)}\right)
\end{aligned}
$$

where $H=H_{1}+H_{2}+T_{1}$. In [16], the above 2-D capacitance model provides accurate capacitance prediction, with a rootmean-square error of $3.68,4.45$, and $16.13 \%$ for $C_{a f}, C_{\text {couple, }}^{1 p}$, and $C_{\text {couple }}^{2 p}$, respectively, compared with the numerical solutions. 


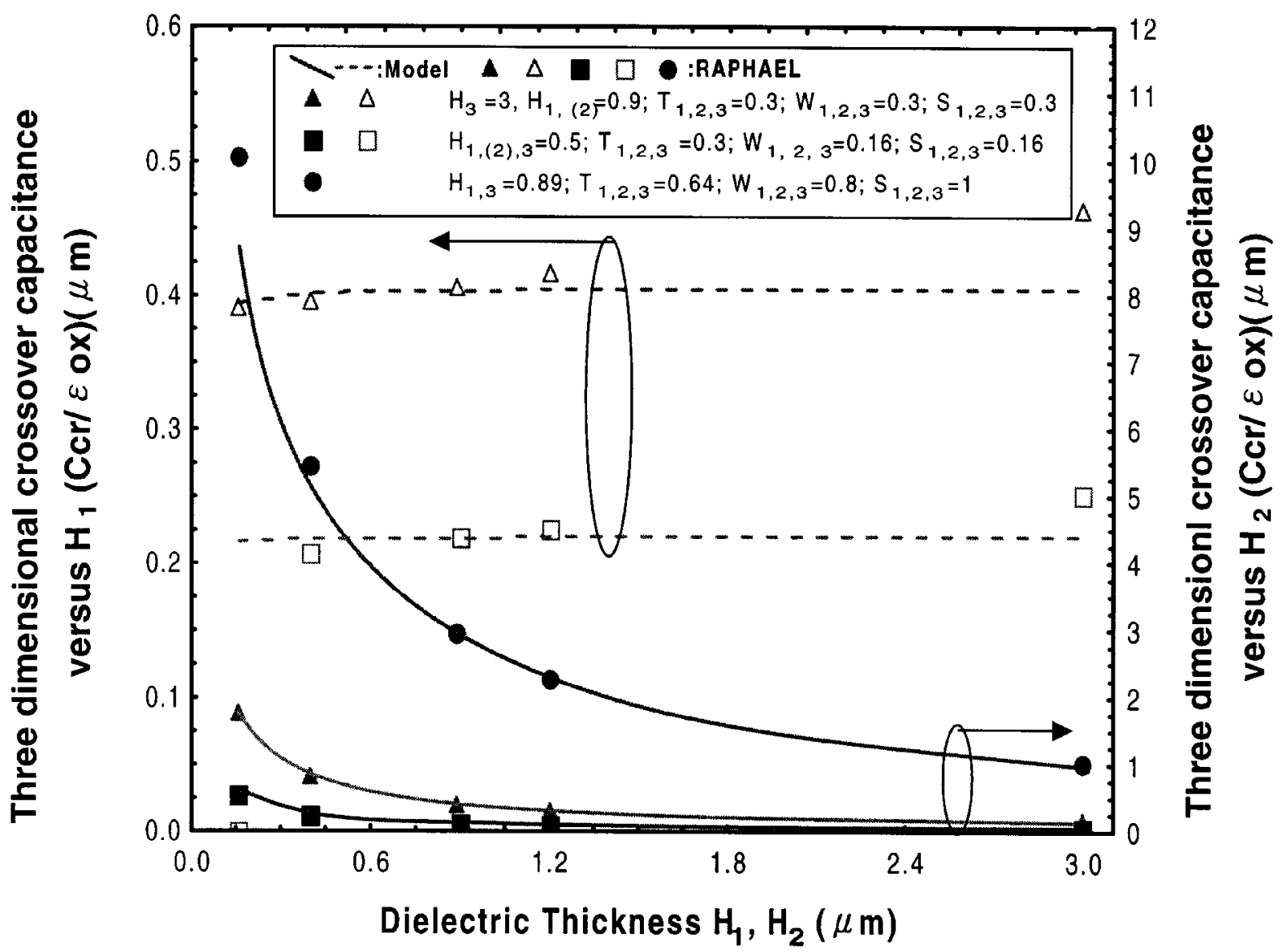

Fig. 2. Crossover capacitance variation versus dielectric thickness. Symbols denote Raphael simulation, and dashed and solid lines denote our model calculation.

The total capacitance on a $M_{2}$ line of length $L$ with $n M_{1}-M_{2}$ crossings and another $n M_{2}-M_{3}$ crossings can be calculated by combining the total intralayer coupling capacitance and the total crossover capacitance. The total intralayer coupling capacitance is easily determined as

$$
\left(L-n W_{1}\right) C_{\text {couple }}^{1 p}+n W_{1} C_{\text {couple }}^{2 p}+\left(L-n W_{1}\right) C_{a f} .
$$

The total crossover capacitance is calculated according to the following: 1) each crossing of $M_{1}$ and $M_{2}$ gives a crossover capacitance $C_{c r\left(M_{1}-M_{2}\right)}$ as exactly calculated by formulas (1), (2), (5), and (6);2) each crossing of $M_{2}$ and $M_{3}$ also gives a crossover capacitance $C_{c r}\left(M_{2}-M_{3}\right)$. To compute $C_{c r\left(M_{2}-M_{3}\right)}$, we need to view the triple-layer upside down before applying the above-developed formulas. That is, $M_{3}$ is now treated as the lower layer and $M_{1}$ the upper layer, which means that $H_{3}$ should be used as $\mathrm{H}_{2}$ and $\mathrm{H}_{2}$ should be used as $\mathrm{H}_{3}$ in the formulas. $\mathrm{H}_{1}$ to be used in the formulas should be the spacing between $M_{3}$ and the next adjacent higher layer (say, $M_{4}$ if exists). If $M_{3}$ is the actual top layer, we have $H_{1}=\infty$. Here, in such a case, we use the value $H_{1}=5 \mu \mathrm{m}$ as infinity.

Combining the crossover and intralayer coupling capacitances, we have

$$
\begin{aligned}
C_{\text {total }}= & n\left[C_{c r\left(M_{1}-M_{2}\right)}+C_{c r\left(M_{2}-M_{3}\right)}\right]+\left(L-n W_{1}\right) C_{\text {couple }}^{1 p} \\
& +n W_{1} C_{\text {couple }}^{2 p}+\left(L-n W_{1}\right) C_{a f} .
\end{aligned}
$$

Our model, which is derived based on three-metal layers, can be applied to a process with any number of metal layers. The crossover capacitance of a metal wire with the layer underneath it can be accurately predicted by our model, with or without above-passing wires. In the general multilayer case, any layer above the first layer or under the third layer is shielded from the second layer and, hence, does not affect the crossover capacitance.

\section{RESULTS AND DisCUSSIONS}

The agreement between our model and the numerical field solver [17] is shown in Table I. In Table I, the error is defined as Error $=($ model-Raphael $/$ Raphael $) \times 100 \%$. The final model has been tested based on 272 data points with a rootmean-square error of $6.71 \%$. The valid ranges of the model are the following: $0.16 \mu \mathrm{m} \leq S_{1}, S_{2}, S_{3} \leq 5 \mu \mathrm{m}, 0.16 \mu \mathrm{m} \leq$ $W_{1}, W_{2}, W_{3} \leq 2 \mu \mathrm{m}, 0.15 \mu \mathrm{m} \leq T_{1}, T_{2}, T_{3} \leq 1.2 \mu \mathrm{m}$, and $0.16 \mu \mathrm{m} \leq H_{1}, H_{2}, H_{3} \leq 3 \mu \mathrm{m}$. The valid ranges for our model were determined based on practical applications in integrated circuit (IC) technology. The upper bound for parameter $S$ set at $5 \mu \mathrm{m}$ is to take care of both dense and sparse lines. The upper bounds for $H$ and $T$ were set to match the practical dieletric and metallization thicknesses.

The comparison between the model and the numerical simulations [17] for various $H_{1}$ and $H_{2}$ is shown in Fig. 2. The strong 


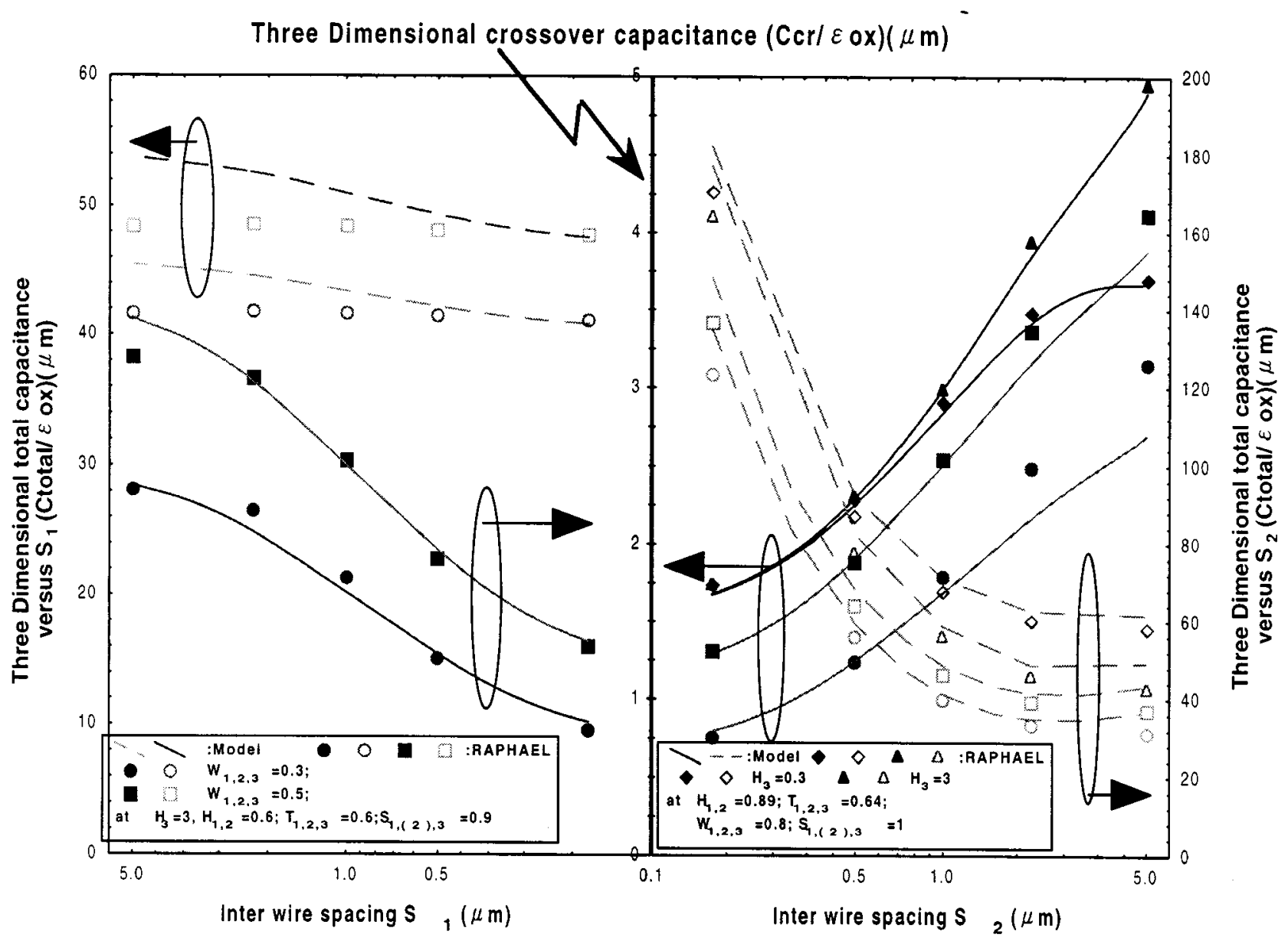

Fig. 3. Crossover capacitance and total capacitance variation versus intralayer wire spacing. Symbols denote Raphael simulation, and lines denote our model calculation. The left graph and right graph are correlated to each other by sharing the common axis of $C_{c r}$ for comparing the different $C_{c r}$ variation versus $S_{1}$ and $S_{2}$.

$H_{2}$ dependence can be accurately predicted by our model. On the other hand, $H_{1}$ only influences $C_{c r}$ minorly, simply because the flux of $C_{c r}$ is shielded from the line-to-ground flux by the intra- $M_{1}$ flux, as shown in the cross-section A in Fig. 1. The intra- $M_{1}$ flux plays the role of electrical buffer between $C_{c r}$ and line-to-ground flux, and this buffer is weakened for small $H_{1}$ (here, $H_{1} \leq 0.5 \mu \mathrm{m}$ ), as shown in Fig. 2. Our model is useful here for predicting strong $H_{2}$ dependence, weak $H_{1}$ dependence induced by intra- $M_{1}$ flux shielding effect, and the onset of weakened shielding effect when $C_{c r}$ reduces with reduced $H_{1}$.

The comparisons of the crossover capacitance $C_{c r}$ and total $M_{2}$ wiring capacitance $C_{\text {total }}$ between our model and the numerical solutions for various intralayer spacing are shown in Fig. 3. Note that $C_{c r}$ decreases with reduced $S_{1}$ and $S_{2}$ because of enhanced intra- $M_{1}$ and intra- $M_{2}$ coupling effects, respectively. At small $S_{1}$ (or $S_{2}$ ), the $C_{c r}$ variation versus $S_{1}$ is symmetrical to that of versus $S_{2}$. At large $S_{1}$ or $S_{2}$, it should be noted that $C_{c r}$ variations with $S_{1}$ and $S_{2}$ are different, and this difference depends on the magnitude of $H_{3}$. To investigate this in more detail, note that for the curves with $H_{3}=3 \mu \mathrm{m}$, $C_{c r}$ increases strongly with increased $S_{2}$ than with increased $S_{1}$, which can be explained as follows: because large $H_{3}($ at $3 \mu \mathrm{m})$ is adopted in these data, increased $S_{2}$ eliminates the intra- $M_{2}$ flux and enhances the $C_{c r}$ flux. On the other hand, with increased $S_{1}$, considerable line-to-ground flux (as shown in the cross-section A of Fig. 1), becomes influential because $H_{1}$ is only $0.6 \mu \mathrm{m}$, and will retard the $C_{c r}$ flux. As a result, $C_{c r}$ increases with increased $S_{2}$ much stronger than with increased $S_{1}$.

Another point worth studying is the following question. When can the impact of top-level metal in any three-level metal combination be ignored for $C_{c r}$ between the first and second level metals? Being able to identify a no-influence region here would allow for an easy $C_{c r}$ estimation without considering parameters of the top-level wire, which will greatly simplify RC extraction [5] and process design [1], [4]. To investigate this process, first it can be observed that the impact of $H_{3}$ is negligible for small $S_{2}$, because $H_{3}=0.3 \mu \mathrm{m}$, and $H_{3}=3 \mu \mathrm{m}$ gives the same $C_{c r}$ for $S_{2} \leq 0.5 \mu \mathrm{m}$, because the strong intra- $M_{2}$ flux completely shields the $M_{3}$-to- $M_{2}$ flux from influencing $C_{c r}$. The impact of $H_{3}$ is much more pronounced when $S_{2}$ is larger than $0.8 \mu \mathrm{m}$, because $C_{c r}$ can be significantly retarded by the $M_{3}$-to- $M_{2}$ flux, as shown in the cross-section B of Fig. 1, especially when the intra- $M_{2}$ coupling disappears. Hence, $C_{c r}$ at $H_{3}=3 \mu \mathrm{m}$ is much larger than at $H_{3}=0.3 \mu \mathrm{m}$. Furthermore, as mentioned before, for $H_{3}=0.3 \mu \mathrm{m}, C_{c r}$ 


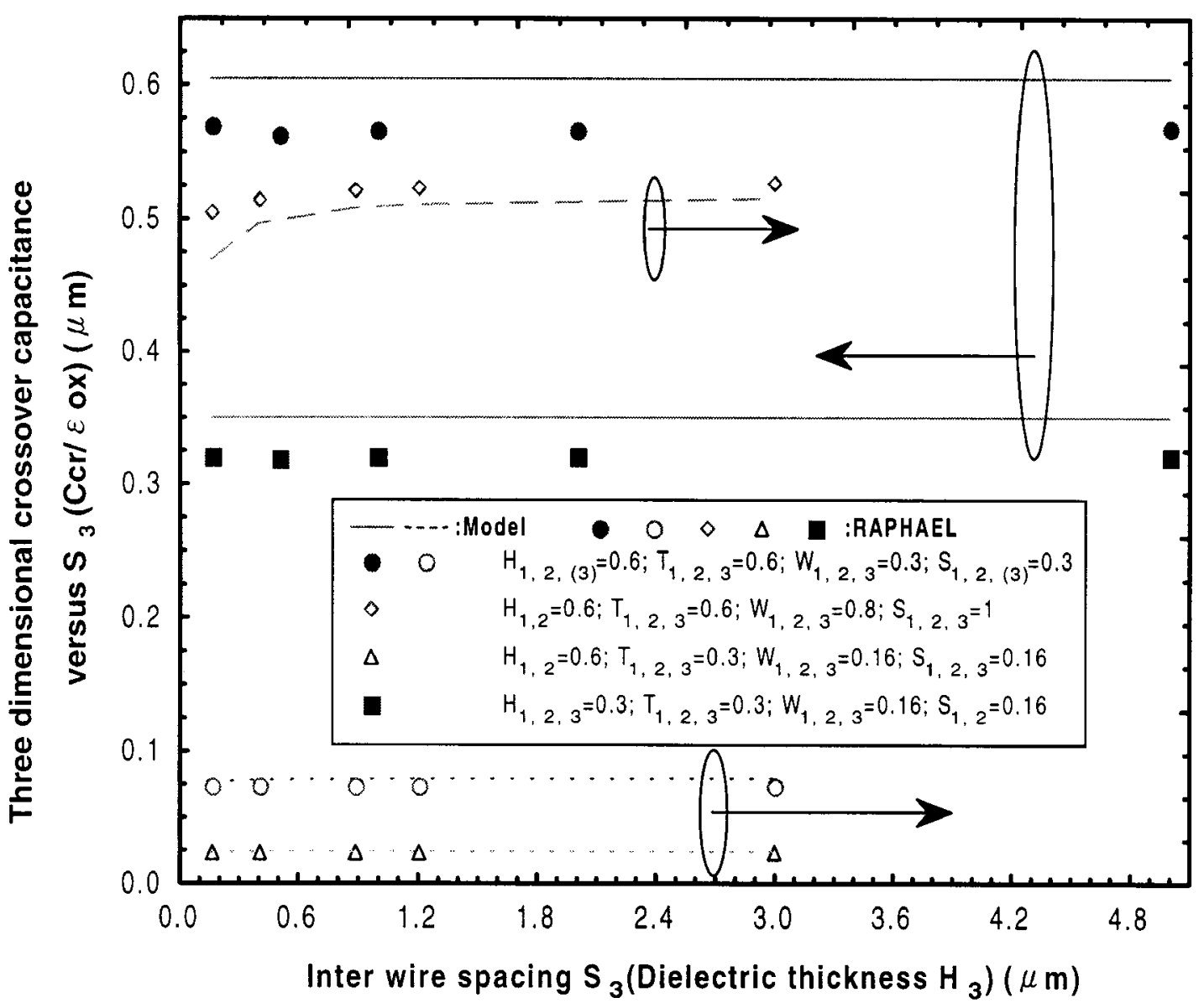

Fig. 4. Crossover capacitance variation versus top-level wire dielectric thickness and intralayer wire spacing. Symbols are Raphael simulation, and lines denote model calculation.

saturates with reduced $H_{3} / S_{2}$ (or increased $S_{2}$ ) for $S_{2} \geq 4 \mu \mathrm{m}$, as predicted by the term $\left(H_{3} /\left(H_{3}+d_{5} S_{2}\right)\right)^{\beta_{5}}$ in (4). These observations on $C_{c r}$ give us a region where $C_{c r}$ is influenced by the third-level metal, and this region is defined by $S_{2} \geq 0.3 \mu \mathrm{m}$ and $H_{3} \leq 0.3 \mu \mathrm{m}$. Outside this region, $C_{c r}$ immunizes from the impact of $M_{3}$, and thus $C_{c r}$ estimation can be performed with the top-level wiring effect ignored.

The calculated $C_{\text {total }}$ is the total $M_{2}$ capacitance in a cell with ten lines for $M_{1}, M_{2}$, and $M_{3}$ each. It is shown that $C_{\text {total }}$ slightly increases with increased $S_{1}$, because of increased $C_{c r}$. On the other hand, $C_{\text {total }}$ significantly decreases with increased $S_{2}$, because of decreased $C_{\text {couple }}^{2 p}$ between $M_{2}$ lines. The larger error of $C_{\text {total }}$ for $S_{1} \geq 0.5 \mu \mathrm{m}$ is induced by the approximation of the last term in (10), i.e., $\left(L-n W_{1}\right) C_{a f}$. The calculation of the 2-D capacitance using this term in the region outside the crossing neighborhood may overestimate the line-to-ground capacitance, because many metal-2 field lines near the crossing neighborhood will be attracted to the crossing metal-1 instead of being terminated to ground, as shown in cross-section A of Fig. 1. This process implies that the approximated length for $C_{a f}$ of $L-n W_{1}$ may cause slight overestimation. Note that because our model mainly tends to be used in deep submicron VLSI, the error for $S_{1}$ larger than $0.5 \mu \mathrm{m}$ may not affect the calculation accuracy for densely packed VLSI.
The impact of the top layer wiring is shown in Fig. 4, where $C_{c r}$ varies with $S_{3}$ and $H_{3} . C_{c r}$ is noticeably reduced with reduced $H_{3}$ when intrawire spacing is $1 \mu \mathrm{m}$, agreeing with our observation from Fig. 3 made in the previous paragraph, as some $M_{2}$ flux is attracted to the $M_{3}$ electrode. The disagreement between the model and numerical solution at small $\mathrm{H}_{3}$ is caused by the large variation generated by the last term in (6) as a result of its rational function form. The form is chosen for tradeoff at large $H_{3}$.

The comparison between our model and measurement data is performed based on test structures fabricated in a $0.35-\mu \mathrm{m}$ twin-well logic CMOS process. The interconnection in this process is composed of $\mathrm{AlCuSi}$ metal lines, an oxide dielectric layer, and chemical-mechanical polished dielectric layers. Three test structures are included in this study, with each test structure composed of three layers of intracoupled wires. Each test structure has 726 crossovers. The $C_{c r}$ between $M_{2}$-to- $M_{3}$ or between $M_{2}$-to- $M_{1}$ is then measured by grounding all additional wires to eliminate all intralayer and line-to-ground flux. Measurement has been performed on ten dies a wafer for four wafers, with the mean and standard deviation shown in Table II. Agreement is shown between our model and measurement with a maximum error of $4.17 \%$. 
TABLE II

COMPARISON BETWEEN MEASUREMENT DATA AND THE MODEL

\begin{tabular}{l|l|l}
\hline $\begin{array}{l}\text { Capacitance/ } \\
\text { Parameters }(\mu \mathrm{m})\end{array}$ & $\begin{array}{l}\text { Model } \\
(\mathrm{aF} / \text { crossover })\end{array}$ & $\begin{array}{l}\text { Measurement } \\
\text { (aF/crossover }) \\
\text { Mean, standard } \\
\text { deviation }\end{array}$ \\
\hline $\mathrm{M}_{2}-\mathrm{M}_{3} \mathrm{C}_{\mathrm{cr}}$ & $24.85,1.64 \%$ \\
$\mathrm{~W}_{1}=\mathrm{W}_{2}=\mathrm{S}_{1}=\mathrm{S}_{2}=0.4$, & 26.06 & \\
$\mathrm{~T}_{1}=\mathrm{T}_{2}=0.6, \mathrm{H}_{1}=2.602$, & & \\
$\mathrm{H}_{2}=0.848, \mathrm{H}_{3}=0.979$ & & $56.49,1.67 \%$ \\
\hline $\mathrm{M}_{2}-\mathrm{M}_{3} \mathrm{C}_{\mathrm{cr}}$ & \\
$\mathrm{W}_{1}=\mathrm{W}_{2}=0.8, \mathrm{~S}_{1}=\mathrm{S}_{2}=0.4$, & & \\
$\mathrm{T}_{1}=\mathrm{T}_{2}=0.6, \mathrm{H}_{1}=2.602$, & & \\
$\mathrm{H}_{2}=0.848, \mathrm{H}_{3}=0.979$ & & \\
\hline $\mathrm{M}_{1}-\mathrm{M}_{2} \mathrm{C}_{\mathrm{cr}}$ & & \\
$\mathrm{W}_{1}=\mathrm{W}_{2}=0.4, \mathrm{~S}_{1}=\mathrm{S}_{2}=0.4$, & & \\
$\mathrm{T}_{1}=\mathrm{T}_{2}=0.6, \mathrm{H}_{1}=0.966$, & & \\
$\mathrm{H}_{2}=0.848, \mathrm{H}_{3}=0.979$ & & \\
\hline
\end{tabular}

It should be noted that our model has been derived based on normalized dielectric constant, and is, hence, independent of the oxide dielectric constant. In comparing our model with measurement data, however, dielectric constant must be determined. The dielectric constant is determined by measuring large-plate capacitors using HP4284 impedance meter at $100 \mathrm{kHz}$, with an exciting signal of $100 \mathrm{mV}$. Based on the measured unit-area capacitance $C_{o x}, \epsilon_{o x}$ is obtained by $\epsilon_{o x}=t_{o x} C_{o x}$. In this work, we have obtained nearly the same $\epsilon_{o x}=3.79$ for all dielectric layers. For cases with various dielectric layers having different values of dielectric constant, it is also possible to apply our model by taking their dielectric constant average as a common $\epsilon_{o x}$ and used in the model equations, but this has not been tested yet.

From our analysis, it is easily seen that the crossover capacitance, because of its 3-D feature, can be influenced by numerous parameters, and the impacts of these parameters are strongly coupled with each other. Our model here can be very helpful in predicting the capacitance variation versus various electrical flux fluctuations, and in optimizing the total capacitance via appropriately adjusting physical dimensions.

\section{CONCLUSION}

A complete closed-form model for the crossover capacitance in multilevel, densely packed interconnections has been developed for arbitrary wiring dimensions. The combined use of the developed crossover capacitance model and existing intralayer coupling and lines-to-ground capacitance model can determine the total capacitance on a wire passing many crossings. The model has been validated by the numerical solutions and measurement data, and it can be used for VLSI design and process optimization.

\section{REFERENCES}

[1] M. T. Bohr, "Interconnect scaling-The real limiter to high performance ULSI," in Proc. IEDM, 1995, pp. 241-244.

[2] J.-H. Chern, J. Huang, L. Arledge, P.-C. Li, and P. Yang, "Multilevel metal capacitance models for CAD design synthesis systems," IEEE Electron Device Lett., vol. 13, pp. 32-34, 1992.
[3] T. Sakurai, "Closed-form formulas for interconnection delay, coupling and crosstalk in VLSI's," IEEE Trans. Electron Device., vol. 40, pp. 118-124, 1993.

[4] K. Rahmat, O. S. Nakagawa, S.-Y. Oh, J. Moll, and W. T. Lynch, "A scaling scheme for interconnect in deep-submicron process," in Proc. IEDM, 1995, pp. 245-248.

[5] N. D. Arora, K. V. Roal, R. Schumann, and L. M. Richardson, "Modeling and extraction of interconnect capacitances for multilayer VLSI circuits," IEEE Trans. Circuits Syst., vol. 15, pp. 58-67, 1996.

[6] K. J. Chang et al., "Parameterized SPICE subcircuits for multilevel interconnect modeling and simulation," IEEE Trans. Circuits Syst.II, vol. 39, pp. 779-789, 1992.

[7] D. H. Cho, Y. S. Eo, M. H. Seung, N. H. Kim, J. K. Wee, O. K. Kwon, and H. S. Park, "Interconnect capacitance, crosstalk and signal delay for $0.35 \mu \mathrm{m}$ CMOS technology," in Proc. IEDM, 1996.

[8] G.-W. Pan, M. Toupikov, and B. K. Gilbert, "A combined finite difference and analytic expression approach to crossover capacitance in a multilayer dielectric environment," IEEE Trans. Comp., Packag., Manufact. Technol. B, vol. 19, pp. 615-620, 1996.

[9] S. A. Kuhn, M. B. Kleiner, P. Ramm, and W. Weber, "Performance modeling of the interconnect structure of a three-dimensional integrated RISC processor/cache system," IEEE Trans. Comp., Packag., Manufact. Technol. B, vol. 19, pp. 719-727, 1996.

[10] V. Vladimir and R. Mittra, "A technique for fast calculation of capacitance matrices of interconnect structures," in IEEE Trans. Comp., Packag., Manufact. Technol. B, vol. 21, 1998, pp. 241-249.

[11] J.-K. Wee, Y.-J. Park, H.-S. Min, D.-H. Cho, M.-H. Seung, and H.-S. Park, "Measurement and characterization of multilayered interconnect capacitance for deep-submicron VLSI technology," IEEE Trans. Semiconduct. Manufact., vol. 11, pp. 636-644, 1998.

[12] P. Nouet and A. Toulouse, "Use of test structures for characterization and modeling of inter-layer capacitances in a CMOS process," IEEE Trans. Semiconduct. Manufact., vol. 10, pp. 233-241, 1997.

[13] K. Aoyama, K. Ise, H. Sato, K. Tsuneno, and H. Mauda, "A new characterization of sub- $\mu \mathrm{m}$ parallel multilevel interconnect and experimental verification," IEEE Trans. Semiconduct. Manufact., vol. 9, pp. 20-26, 1996.

[14] C.-J. Chao, S.-C. Wong, M.-J. Chen, and B. K. Liew, "An extraction method to determine interconnect parasitic parameters," IEEE Trans. Semiconduct. Manufact., vol. 11, pp. 615-623, 1998.

[15] J. C. Chen, D. Sylvster, and C. Hu, "An on-chip interconnect capacitance characterization method with sub-femto-farad resolution," IEEE Trans. Semiconduct. Manufact., vol. 11, pp. 204-210, 1998.

[16] S.-C. Wong, G.-Y. Lee, and D.-J. Ma, "Modeling of interconnect capacitance, delay and cross-talk in VLSI," IEEE Trans. Semiconduct. Manufact., to be published.

[17] RAPHAEL Users' Manual, Technology Modeling Associate, 1995.

[18] J. D. Kraus and K. R. Carver, Electromagnetics, 2nd ed. New York: McGraw-Hill, 1973, pp. 290-304.

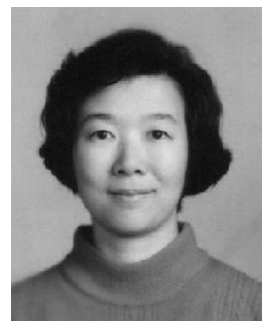

Shyh-Chyi Wong received the B.S. degree in electronics engineering from the National Chiao-Tung University, Taiwan, R.O.C., in 1983 and the M.S. and Ph.D. degrees in electrical engineering from the University of Maryland, College Park, in 1985 and 1989 , respectively.

From 1989 to 1993, she was with Analog Devices Inc., MA, where she worked on SPICE modeling and characterization of various MOS devices, circuits, and processes. From 1993 to 1995 , she was with Taiwan Semiconductor Manufacturing Corp. as the Manager of the Device Department. From 1995 to 1998, she was with the Department of Electronics Engineering, Feng-Chia University, Taiwan, as an Associate Professor. From 1998 to 1999, she was with the Winbond Electronics Corp., Taiwan, as the Director of Device and Reliability Division. Since 1999, she has been with Taiwan Semiconductor Manufacturing Corp. as the Program Manager for mixed signal and RF processes. Her research interests include device modeling and simulation, interconnect modeling and layout extraction, statistical modeling, device reliability, and analog circuit design. 


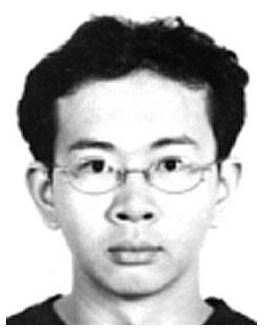

Trent Gwo-Yann Lee was born in Taiwan in 1974. He received the B.S. and M.S. degrees in electrical engineering from Feng-Chia University in 1996 and 1998 , respectively. He is currently pursuing a Ph.D. degree from National Chiao-Tung University.

His research interests include interconnection and device modeling and computer simulation method.

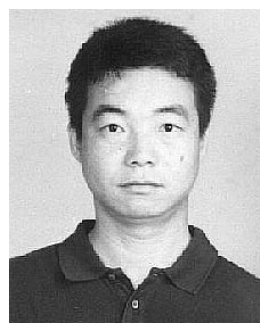

Dye-Jyun Ma received the B.S. degree in electronics engineering from National Chiao-Tung University, Taiwan, R.O.C., in 1979 and the M.S. and Ph.D. degrees in electrical engineering from the University of Maryland, College Park, in 1984 and 1988, respectively.

From May 1988 to June 1993, he was with Digital Equipment Corp., MA, as a Principal Engineer. Since August 1993, he has been with the Department of Electrical Engineering, National Chung-Hsing University, where he is presently a Professor. His research interests include control and optimization and interconnect and statistical modeling.

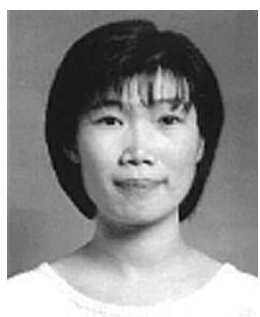

Chuan-Jane Chao received the B.S. and M.S. degrees from National Chiao-Tung University (NCTU), Hsinchu, Taiwan, R.O.C., in 1990 and 1992, respectively, all in electronics engineering. She is currently pursuing the $\mathrm{Ph} . \mathrm{D}$. degree in electronics engineering at NCTU.

From 1992 to 1998, she was with Taiwan Semiconductor Manufacturing Corp., Hsinchu, Taiwan, R.O.C., as a Principle Device Engineer in device engineering, responsible for SPICE modeling and device characterization. Since October 1998, she has been with Winbond Electronics Corp., Hsinchu, where she works on SPICE modeling and characterization of various MOS devices, circuits, and processes. Her research interests are in the area of device and interconnect modeling and characterization. 